\title{
Aberrations of the Human Eye in Visible and Near Infrared Illumination
}

\author{
LOURDES LLORENTE, OD, LUIS DIAZ-SANTANA, PhD, DAVID LARA-SAUCEDO, BSc, and \\ SUSANA MARCOS, PhD
}

\begin{abstract}
Instituto de Óptica "Daza de Valdés", Consejo Superior de Investigaciones Cientificas, Serrano, Madrid, Spain (LL, SM), Applied Vision Research Centre, Department of Optometry and Visual Science, City University, Northhampton Square, London, United Kingdom (LDS), Blackett Laboratory, Imperial College of Science Technology and Medicine, London, United Kingdom (DLS)
\end{abstract}

\begin{abstract}
Purpose. In most current aberrometers, near infrared light is used to measure ocular aberrations, whereas in some applications, optical aberration data in the visible range are required. We compared optical aberration measurements using infrared $(787 \mathrm{~nm})$ and visible light $(543 \mathrm{~nm})$ in a heterogeneous group of subjects to assess whether aberrations are similar in both wavelengths and to estimate experimentally the ocular chromatic focus shift. Methods. Ocular aberrations were measured in near infrared and visible light using two different laboratory-developed systems: laser ray tracing (LRT) and Shack-Hartmann. Measurements were conducted on 36 eyes (25 and 11 eyes, respectively), within a wide range of ages ( 20 to 71 years), refractive errors $(-6.00$ to +16.50$)$, and optical quality (root mean square wavefront error, excluding defocus, from 0.40 to $9.89 \mu \mathrm{m}$ ). In both systems, wave aberrations were computed from the ray aberrations by modal fitting to a Zernike polynomial base (up to seventh order in laser ray tracing and sixth order in Shack-Hartmann). We compared the Zernike coefficients and the root mean square wavefront error corresponding to different terms between infrared and green illumination. Results. A Student's t-test performed on the Zernike coefficients indicates that defocus was significantly different in all of the subjects but one. Average focus shift found between $787 \mathrm{~nm}$ and $543 \mathrm{~nm}$ was $0.72 \mathrm{D}$. A very small percentage of the remaining coefficients was found to be significantly different: $4.7 \%$ of the 825 coefficients ( 25 eyes with 33 terms) for laser ray tracing and $18.2 \%$ of the 275 coefficients (11 eyes with 25 terms) for Shack-Hartmann. Astigmatism was statistically different in $8.3 \%$ of the eyes, root mean square wavefront error for third-order aberrations in $16.6 \%$, and spherical aberration $\left(\mathrm{Z}_{4}{ }^{0}\right)$ in $11.1 \%$. Conclusions. Aerial images captured using infrared and green light showed noticeable differences. Apart from defocus, this did not affect centroid computations because within the variability of the techniques, estimates of aberrations with infrared were equivalent to those measured with green. In normal eyes, the Longitudinal Chromatic Aberration of the Indiana Chromatic Eye Model can predict the defocus term changes measured experimentally, although the intersubject variability could not be neglected. The largest deviations from the prediction were found on an aphakic eye and on the oldest subject. (Optom Vis Sci 2003;80:26-35)
\end{abstract}

Key Words: ocular aberrations, Shack-Hartmann, laser ray tracing, near infrared

$\mathrm{n}$ the last few years, there has been a renewed interest in the measurement and understanding of the aberrations of the human eye. Along with studies addressing important basic questions on physiological optics (i.e., change of aberrations with accommodation, ${ }^{1,2}$ age, ${ }^{3,4}$ retinal eccentricity, ${ }^{5}$ refractive error ${ }^{6,7}$ ), clinical applications of aberrometry are rapidly increasing. For example, it has been shown to be a useful tool in assessing keratoconus ${ }^{8,9}$ or corneal transplantation. ${ }^{10,11}$ In particular, aberrometry is of great use in refractive surgery, both as a tool to assess the outcomes of refractive surgery ${ }^{12-15}$ and as a guide to optimize ablation algorithms to eventually compensate for the ocular aberrations. ${ }^{16}$ In addition, static ${ }^{17}$ or dynamic aberration correction, ${ }^{18,19}$ with great potential for high-resolution ophthalmoscopy, ${ }^{20}$ relies on the accurate measurement of aberrations.

All aberrometers are based on the common principle of measuring the slopes of the wave aberration, either as a light enters the eye (i.e., laser ray tracing, ${ }^{21,22}$ spatially resolved refractometer, ${ }^{23,} 24$ Tscherning aberrometer ${ }^{25}$ or the crossed-cylinder aberroscope ${ }^{26}$ ) or as it emerges from the eye (Shack-Hartmann ocular wavefront sensor ${ }^{27,28}$ ). Apart from the spatially resolved refractometer, 
which is a psychophysical technique (and therefore visible light must be used) the rest of these techniques measure the light reflected by the retina. Most of the currently available wavefrontsensing techniques use infrared (IR) illumination, which has several advantages over visible light. It is more comfortable for the patient because the human eye is less sensitive to $\mathrm{IR}^{29}$; pupil dilation is not strictly required; the retina reflects a higher percentage of the incident light compared with shorter wavelengths ${ }^{30}$; and backscatter by the anterior optics ${ }^{31}$ is reduced. Dynamic measurement of aberrations is then possible using IR illumination ${ }^{32}$ with natural accommodation because mydriasis (and its associated cycloplegic effects) is not necessary.

Although current aberration measurements are typically done with IR light, in most applications, data from visible light are required. For direct comparison between optical measurements (estimated from the wave aberration) and visual performance we need to make sure that the results obtained in IR light are equivalent to those obtained with visible light. This is particularly important if the measured wave aberration is planned to be used to guide ablation in refractive surgery procedures, where the aim is to improve the patient's visual performance. Knowledge of the defocus shift between IR and visible wavelengths is essential if the results are to be used to predict refraction.

Previous measurements of aberrations at different visible wavelengths using a spatially resolved refractometer showed slight differences in some aberration terms as a function of wavelength. ${ }^{33}$ The chromatic difference of focus agreed with previous psychophysical results from the literature; however, the longitudinal chromatic aberration (LCA) based on reflectometric double-pass measurements ${ }^{34,35}$ has been reported to be lower than conventional psychophysical estimates. These results prompted us to revisit the question of whether reflections at different retinal layers may be the cause for the discrepancy. The following questions hence arise: (1) Are the aberrations measured with IR and green light equivalent? (2) Is the focus difference between IR and green predictable by the LCA (and therefore reasonably predictable across subjects), or can the relative differences in reflectance and scattering across wavelengths be affecting the aberration measurements?

There are two previous studies that compare visible and near infrared optical quality in the human eye. ${ }^{36,37}$ Double-pass measurements of modulation transfer functions in IR and green light appear to be similar. In this previous study, subtraction of background halos (noticeably different between IR and green) was critical. ${ }^{36}$ The other study used an objective crossed-cylinder aberroscope to measure aberrations and reported that aberrations are virtually identical in near IR and green light. ${ }^{37}$ However, the data analysis was mainly qualitative and limited to three eyes.

In this paper, we compare ocular aberrations between near IR (786 $\mathrm{nm}$ for laser ray tracing [LRT] and $788 \mathrm{~nm}$ for Shack-Hartmann $[\mathrm{SH}])$ and visible illumination $(543 \mathrm{~nm})$ measured with two objective techniques, LRT and SH. These are experimental systems developed at Instituto de Optica (CSIC), Madrid, Spain and Imperial College, London, UK, respectively, but the conclusions drawn here can be extrapolated to recent unrelated commercially available instruments, based on similar principles. We performed measurements on 36 subjects with a wide range of ages, refractions, and ocular conditions (including old and surgical eyes), thus cov- ering a wide range of aberrations and, potentially, ocular and retinal structural differences.

\section{METHODS Laser Ray Tracing}

Setup and Procedures. The laser ray tracing technique (Fig. 1A) has been described in detail elsewhere. ${ }^{5,13,21,22,38} \mathrm{~A}$ set of 37 parallel laser pencils sequentially scans a $6.51-\mathrm{mm}$ pupil in a $1-\mathrm{mm}$ step-hexagonal pattern. Aerial images formed by the light reflected off the retina are simultaneously recorded on a high-resolution

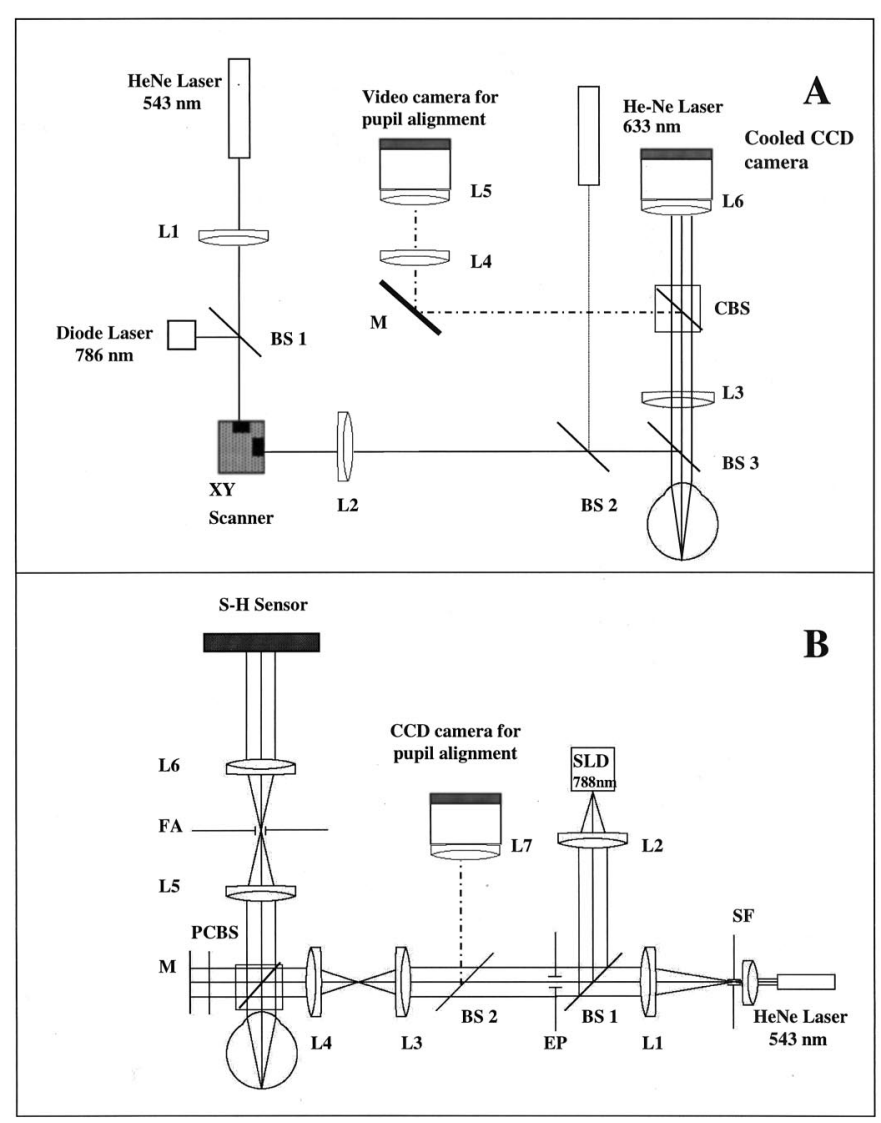

FIGURE 1.

Schematic diagrams of the laser ray tracing (A) and Shack-Hartmann wavefront sensor (B) setups. A: In laser ray tracing, a laser beam from a He-Ne $(543 \mathrm{~nm})$ laser or a diode laser $(786 \mathrm{~nm})$ samples the pupil plane by means of an XY scanner and collimating lens L1. Light reflected off the retina forms an aerial image onto a cooled CCD camera by means of the lens L3 and camera objective L6. A red He-Ne laser $(633 \mathrm{~nm})$ acts as a fixation point. A video camera, conjugate to the pupil by means of lens L4 and video camera objective L5 monitors pupil centration. BS1 and BS2 are pellicle beam splitters, BS3 is a glass beam splitter, CBS is a cube beam splitter and $M$ is a mirror. B: In Shack-Hartmann, light coming from an expanded $\mathrm{He}-\mathrm{Ne}(543 \mathrm{~nm})$ laser or from a super luminescent diode (SLD) forms a point on the retina. SF is a spatial filter, and L1 and L2 are collimating lenses. L3, L4 and L5, L6 are relay systems in the illumination and imaging channels, respectively. EP is an entry pupil aperture (pupil diameter $=1.5 \mathrm{~mm}$ ), and FA is a field aperture. Light reflected off the retina is imaged by a Shack-Hartmann sensor (S-H Sensor) on a cooled CCD camera. Images of the pupil are projected onto a CCD camera by objective lens L7 and monitors pupil centration. BS1 and BS2 are pellicle beam splitters, and PCBS is a polarizing cube beam splitter. $M$ is a mirror that serves in reference image capture. 
CCD camera. The centroid of each aerial image is estimated. The deviations of the centroids from the reference (which is the position of the centroid corresponding to the chief ray) are proportional to the local derivative of the wave aberration. The wave aberration is obtained from the sets of derivatives by means of a modal fitting to the Zernike polynomial basis (through seventh order). In previous studies using this technique, measurements were obtained using a 543-nm HeNe laser beam (Melles Griot, 5 $\mathrm{mW})$. For this study, light from an IR $(786 \mathrm{~nm})$ laser diode coupled to an optical fiber (Schäfter + Kirchhoff, $15 \mathrm{~mW}$ ) was inserted into the system using a pellicle beam splitter and coaligned to the green beam. Both lasers were attenuated, by means of neutral-density filters, and light exposure was at least one order of magnitude below safety limits. ${ }^{39}$

Setting and Control Experiment. Measurements were conducted at Instituto de Óptica, CSIC, Madrid, Spain. The system was calibrated to verify that it did not introduce chromatic aberration. For this purpose, we placed a calibrated aberrated phase plate $^{17}$ in front of a diffraction-limited artificial eye and measured its aberrations using green and IR light. Identical results were obtained for all aberration terms within the accuracy of the technique, including the defocus term, and replicated the nominal aberrations of the phase-plate.

Subjects. We measured 25 eyes (labeled \#1 to \#25) from 16 subjects: 19 eyes were normal, one eye was aphakic (\#8), and five eyes had undergone laser-assisted in situ keratomileuesis refractive surgery (\#5, \#6, \#10,\#12, and \#13). Ages ranged from 20 to 71 years (mean $\pm S D, 33 \pm 11$ years), spherical error ranged from -6.00 to $+16.50 \mathrm{D}$ (mean, $-1.62 \pm 4.42 \mathrm{D}$ ), and astigmatism ranged from 3.78 to $0.07 \mathrm{D}$ (mean, $1.07 \pm 0.98 \mathrm{D}$ ).

Before the measurement, an informed consent form approved by institutional ethical committees was signed by each patient in accordance with the tenets of the Declaration of Helsinki. The pupil was dilated with one drop of Tropicamide $1 \%$.

Measurements. Subjects were stabilized with a dental impression and a forehead rest, and the eye was monitored with a CCD camera to ensure alignment of the pupil center to the optical axis of the instrument during the measurement. Spherical refractive errors were compensated with trial lenses when necessary.

Each session consisted of 10 runs, each run (37 images corresponding to the 37 rays sampling the pupil) lasted approximately $4 \mathrm{~s}$. Five consecutive series were collected using green light (543 $\mathrm{nm}$ ), and then five series were collected using near IR light (786 $\mathrm{nm})$.

\section{Shack-Hartmann}

Setup and Procedures. A schematic diagram of the $\mathrm{SH}$ wavefront sensor used in this study is shown in Fig. 1B. A detailed description of a similar system can be found elsewhere $38,40-42$ without the minor modifications introduced for this study. Light from an IR $(788 \mathrm{~nm})$ super luminescent diode (Anritsu, $10 \mu \mathrm{W}$ ) was introduced by means of a pellicle beam splitter and coaligned to the green $(543 \mathrm{~nm}) \mathrm{He}-\mathrm{Ne}$ laser beam (Melles Griot, $1 \mathrm{~mW}$ ) used in previous measurements. The He-Ne laser was spatially filtered and expanded before collimation, bringing the maximum power reaching the eye to $<5 \mu \mathrm{W}$ over an 8 -mm diameter pupil. Further power reduction was achieved by reducing the beam di- ameter to 1.5 to $2 \mathrm{~mm}$ and by the use of neutral-density filters before spatial filtering. The super luminescent diode power was largely reduced after fiber coupling (to about $10 \%$ of its maximum nominal power), and further power reduction was electronically controlled with its driver. In all cases, the maximum power reaching the eye was at least one order of magnitude below the safety limits. ${ }^{39}$ The principle of the $\mathrm{SH}$ system has been described extensively in the literature. A narrow collimated laser beam forms a spot on the retina, and the light reflected and emerging from the eye is sampled by a rectangular lenslet array placed on a plane conjugate to the eye pupil. A CCD camera, placed on the focal plane of the lenslet array and conjugate to the retina, is used to record the $\mathrm{SH}$ spot pattern. Deviations from the ideal SH spot pattern are proportional to the local slopes of the wave aberration.

For this study, wave aberration was estimated from measured slopes using a least-mean square procedure. Wave aberration was fitted to a sixth-order Zernike polynomial expansion (27 terms).

The size of each lenslet was $0.8 \mathrm{~mm} \times 0.8 \mathrm{~mm}$ over the pupil plane, and the focal length was $35 \mathrm{~mm}$. The pupil size was $6 \mathrm{~mm}$.

Setting and Control Experiment. Measurements were conducted at Imperial College of Science Technology and Medicine, London, United Kingdom. The system was calibrated to ensure that it did not introduce chromatic aberration. Two reference $\mathrm{SH}$ images using green and IR light were compared. The green reference was used to calculate the aberrations of the IR reference. The order of magnitude of every Zernike coefficient was always smaller than or equal to the standard deviations of any series of 10 measurements of ocular aberrations using only one wavelength. This procedure proves that no significant amount of chromatic aberration is introduced by the optics of the system.

Subjects. We measured 11 normal eyes (labeled \#26 to \#36) (six subjects). Ages ranged from 22 to 26 years (mean, $23 \pm 1.47$ years), spherical error ranged from -6.00 to $+0.75 \mathrm{D}$ (mean, 2.51 $\pm 3.24 \mathrm{D}$ ), and astigmatism ranged from 0.07 to 4.00 (mean, 1.30 $\pm 1.5 \mathrm{D})$.

The institutional research and ethical committee approved the use of the wavefront sensor and the experimental design. Written consent was obtained from all subjects participating in the study according to the tenets of the Declaration of Helsinki. Pupils were dilated using Tropicamide 1\% and Phenylephrine 2.5\% $30 \mathrm{~min}$ before the beginning of the measurements.

Measurements. Subjects were stabilized with the help of a dental impression, and the pupil of the eye was aligned to the optical axis of the instrument while it was continuously monitored with a CCD camera. The illumination source was used as the fixation point. Spherocylindrical refractive errors were compensated when necessary.

At least six series of $10 \mathrm{SH}$ images were collected, three using green illumination $(543 \mathrm{~nm})$ and the rest using IR illumination $(788 \mathrm{~nm})$. Images with the same wavelength were collected consecutively.

\section{Equivalence of LRT and SH}

The equivalence of the SH and LRT wavefront sensors has been demonstrated in previous studies. ${ }^{22,43}$ Control measurements on two subjects showed that the SH and LRT systems used in this 
study (in Madrid and London, respectively) provided similar aberrations in normal eyes. ${ }^{38}$

\section{RESULTS \\ Raw Data}

Raw data obtained from both techniques consist of a set of aerial images (in different frames for the LRT or a single frame for the $\mathrm{SH}$ ). Each image corresponds to a pupil position (entry pupil position for LRT and exit pupil position for $\mathrm{SH}$ ).

Fig. $2 \mathrm{~A}$ and $\mathrm{B}$ shows a set of aerial images obtained with LRT for eye \#5, for green and IR light, respectively. Each image has been placed at the corresponding entry pupil position. The intensity patterns differ significantly across wavelengths. Fig. 2C shows the spot diagram (joint plot of the position of the centroids of the same set) corresponding to the average data of three consecutive runs with green light (crosses) and four consecutive runs with IR light (circles) for eye \#5. The error bars indicate the standard deviation of the positions of the centroid between runs. Chromatic defocus is responsible for the consistent shift between wavelengths, which increases with entry pupil eccentricity.

Fig. $2 \mathrm{D}$ and $\mathrm{E}$ shows $\mathrm{SH}$ images for green and IR light, respectively, for eye \#29. The presence of a halo surrounding the centroid is more evident for the image with IR illumination than for that with green illumination. The spots at the upper right and the lower left corners of the image appear dimmer (particularly for green illumination) due to the use of crossed polarization between illu- mination and recording. ${ }^{38}$ Fig. 2F shows the $\mathrm{SH}$ centroids corresponding to D (crosses) and E (circles). As in LRT, the shift between the green and IR spots increases toward the periphery of the image.

\section{Wave Aberration Maps}

Fig. 3 shows wave aberration maps from LRT measurements for both wavelengths for third- and higher-order aberrations. Eyes \#9 and \#22 were normal eyes, whereas \#13 had undergone laserassisted in situ keratomileuesis surgery. Each map is the average of at least three experimental runs. Contour lines have been plotted every $0.2 \mu \mathrm{m}$. Fig. 4 shows wave aberration maps for three normal eyes (\#29, \#30, and \#31) measured with SH for both wavelengths, excluding tilt and defocus. Contour lines have been plotted every $0.5 \mu \mathrm{m}$

For both systems, the wave aberration patterns corresponding to green and IR wavelengths for the same subject are very similar.

\section{Zernike Coefficients and Root Mean Square Wavefront Error (RMS)}

Fig. 5 shows plots of sets of Zernike coefficients for green (crosses) and IR (circles) light for the same eyes as in Figs. 3 and 4. The coefficient ordering and normalization follows the Optical Society of America standardization committee recommendations. ${ }^{44}$ First-

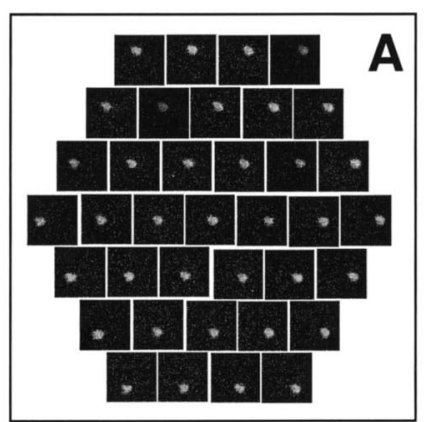

$543 \mathrm{~nm}$

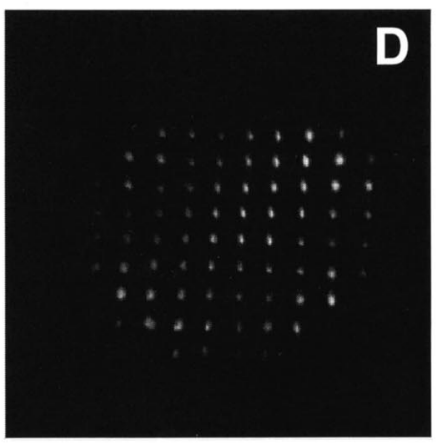

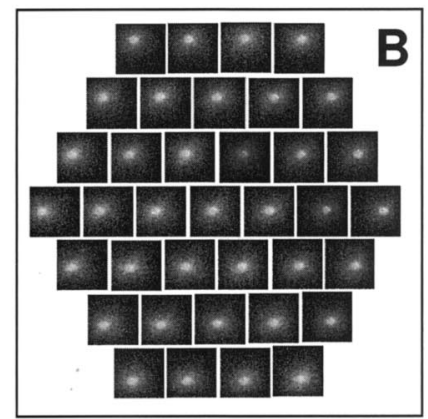

$786 \mathrm{~nm}$

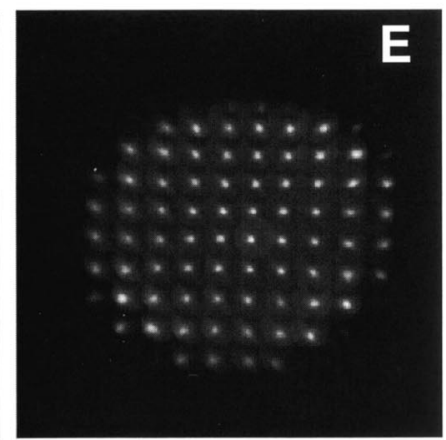

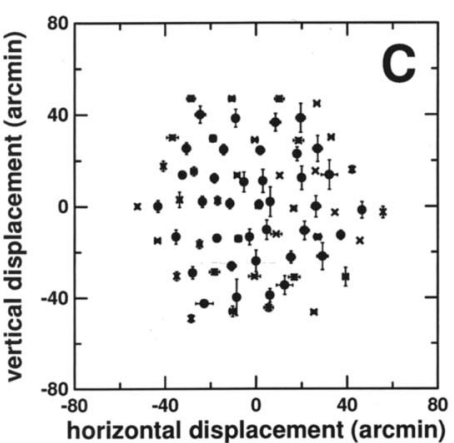

$X$ Green Infrared

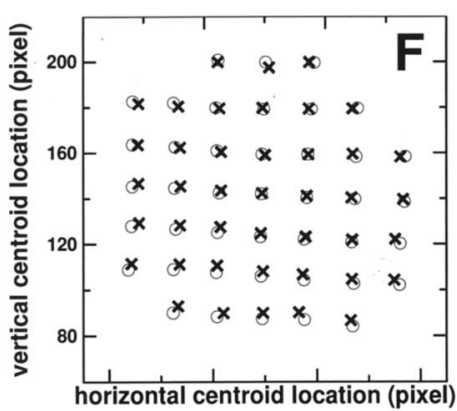

\section{FIGURE 2.}

Raw data as obtained from laser ray tracing (panels A, B, and C) and Shack-Hartmann wavefront sensor (panels D, E, and F). In laser ray tracing, a series of retinal images is captured sequentially as a function of the entry pupil position. Aerial images obtained for eye $\# 5$ using green and infrared light are shown in panels $A$ and B, respectively. Panel $C$ shows the corresponding spot diagram. Crosses represent green illumination, and circles represent IR illumination. Panels D and E show Shack-Hartmann images for eye \#29 for green and IR light, respectively. Panel E plots the corresponding centroids of the $\mathrm{SH}$ images. Symbol notation is the same as for panel C. 
and second-order terms have been cancelled to allow a higherresolution view of higher-order terms.

Error bars represent the standard deviation of the measurement. Mean variability (standard deviation) averaged across Zernike coefficients and subjects was $0.10 \pm 0.06$ for green light and $0.07 \pm$ 0.04 for IR light for the measurements performed with LRT and $0.019 \pm 0.009$ for green light and $0.015 \pm 0.009$ for IR light for the measurements performed with SH. The differences between the Zernike coefficients measured with green or IR light shown in Fig. 6 are within the inherent variability of the techniques.

We performed a univariate statistical analysis (Student's t-test)

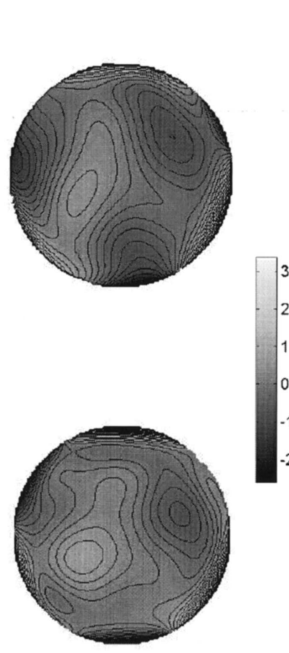

Eye \#9

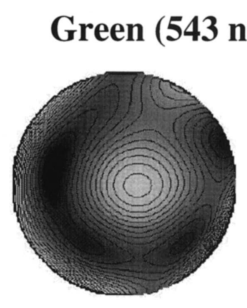

Eye \#13

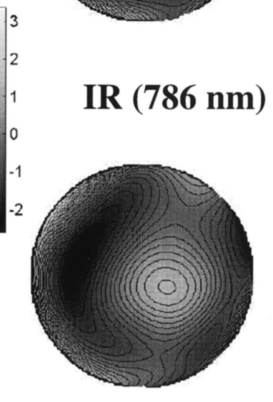

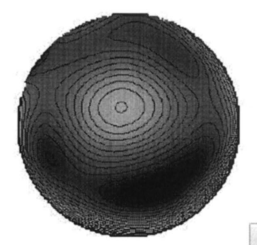

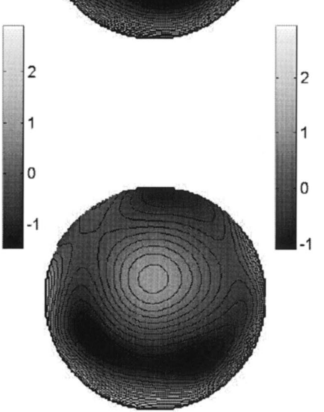

Eye \# 22

\section{FIGURE 3.}

Wave aberration maps from laser ray tracing measurements for green and infrared light. First- and second-order terms have been excluded. Eyes \#9 and $\# 22$ were normal eyes, and \#13 had undergone laser-assisted in situ keratomileusis surgery. Each map is the average of at least three experimental runs. Contour lines are plotted every $0.2 \mu \mathrm{m}$.

\section{Green $(543$ nm)}

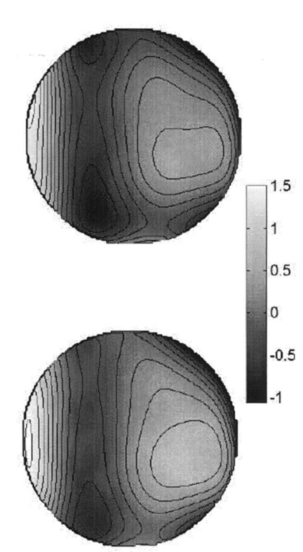

Eye \# 30

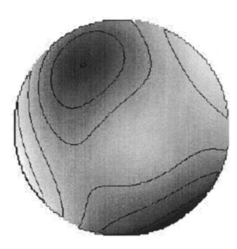

IR (786 nm)

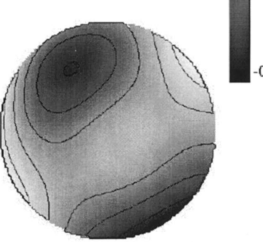

Eye \# 29

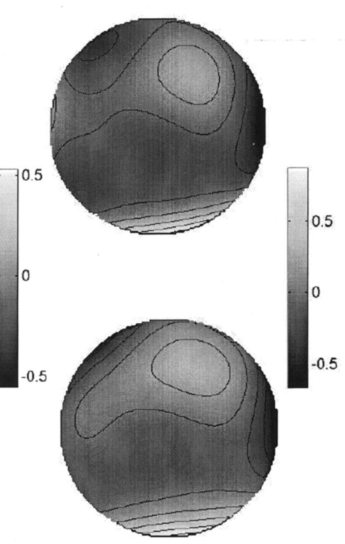

Eye \# 31

\section{FIGURE 4.}

Wave aberration maps for three normal eyes $(\# 29$, $\# 30$, and $\# 31$ ) measured with a Shack-Hartmann wavefront sensor for both wavelengths. Tilts and defocus have been excluded. Contour lines are plotted every $0.5 \mu \mathrm{m}$.
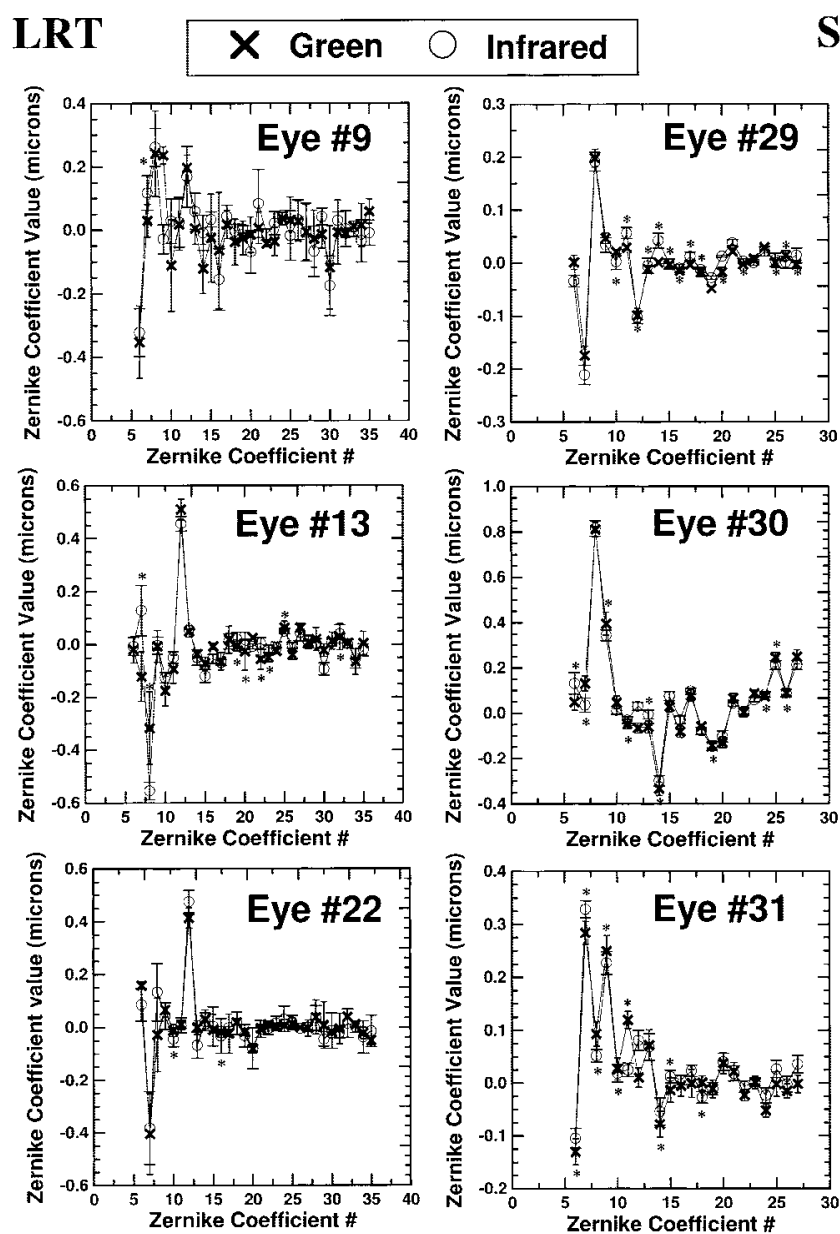

FIGURE 5.

Plots of sets of the Zernike coefficients for green (crosses) and infrared (circles) light for the same eyes as in Fig. 3 and 4. The coefficient ordering and normalization follows the Optical Society of America standardization committee recommendations. ${ }^{45}$ First- and second-order terms have been cancelled. Error bars represent the standard deviation of the measurement. Asterisks indicate the statistically different coefficients.

on each Zernike coefficient for each eye to detect which subjects and particular terms showed significant differences $(\mathrm{p}<0.01)$ when measured in green and IR light. For the 25 eyes measured with LRT only 39 coefficients (excluding defocus) of $825(25 \times 33$ terms), i.e., $4.7 \%$, were statistically different. The defocus term $\left(Z_{0}^{2}\right)$ was statistically different in 24 of the 25 eyes $(96 \%$ of the subjects). All the other statistically different terms were randomly distributed.

For the 11 eyes measured with SH, 22\% (61 of 275 [ 25 coefficients $\times 11$ eyes]) of the coefficients were statistically different. The defocus term $\left(Z_{0}^{2}\right)$ was statistically different in all of the eyes. Among terms other than defocus, 50 of 275 (18\%) were found to be significantly different.

Fig. 6 shows defocus for IR wavelength vs. green wavelength in diopters for all subjects. There is a good linear correlation $\left(\mathrm{R}^{2}=\right.$ 0.976), and the slope of the linear fit is close to one (0.9615). The focus shift between IR and green given by the fitting equation is 0.722 . The experimental focus shift was $0.78 \pm 0.29 \mathrm{D}$.

Bar diagrams in Fig. 7 compare individual terms (astigmatism and spherical aberration) and the RMS including different terms 


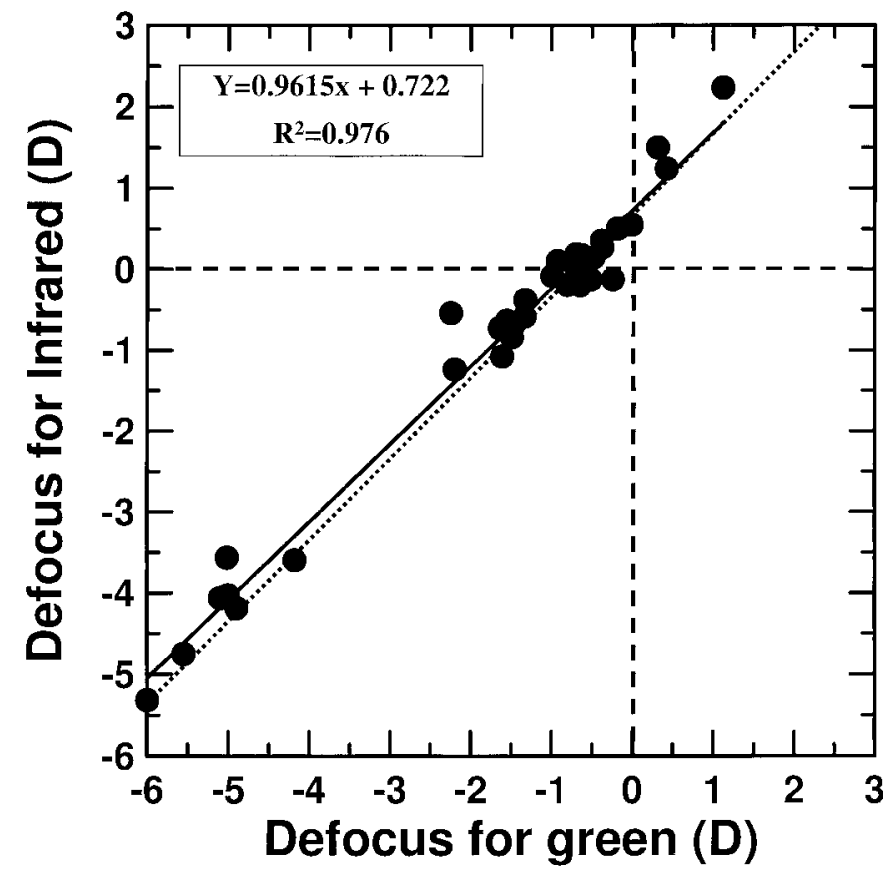

FIGURE 6.

Defocus for infrared vs. green wavelength in diopters for all subjects. The solid line represents the best linear fit to the data $\left(R^{2}=0.976\right)$. The focus shift between infrared and green given by the fitting equation is $0.722 \mathrm{D}$. The slope of the linear fit is close to one (0.9615). The dashed line corresponds to a fitting line with slope equal to one and falls within the data variability.

obtained with green (black bars) and IR (gray bars) wavelengths for all subjects. Eyes \#1 to \#25 were measured with LRT, and eyes \#26 to \#36 were measured with SH. Asterisks indicate eyes that showed statistically significant differences $(\mathrm{p}<0.01)$. Astigmatism (Fig. $7 A)$ was statistically different in three of the 36 subjects (8.3\%). RMS for third-order aberrations (Fig. 7B) was statistically different in six of the 36 subjects (16.6\%). RMS for third-and higher-order aberrations (Fig. 7C) was statistically different in five of the 36 subjects (13.5\%). Spherical aberration $\left(Z_{4}^{0}\right)$ (Fig. 7D) was statistically different in four of the 36 subjects $(11.1 \%)$

Only one eye (\#35) came out significantly different for all the terms or orders reported above (RMS for third- and higher-order aberrations, third-order aberrations, spherical aberration, and astigmatism).

\section{DISCUSSION}

This study shows that although the intensity distribution of LRT aerial images or SH images is notably different between green and IR illumination, both wavelengths provide aberration estimates within the experimental error (except for defocus). Our sample includes eyes with large differences in optical quality (from normal eyes to surgical eyes) and ages (20 to 71 years), suggesting that this conclusion holds for most of the population.

\section{Differences in Image Intensity Profiles}

Fig. 2 shows relevant intensity differences between the aerial images obtained with IR and those obtained with green illumina- tion. IR images are typically more spread and are surrounded by a broad halo. It has been suggested that most of the light contributing to the core of double-pass aerial images is probably due to the light captured and guided back from the photoreceptors. ${ }^{36,45}$ The halo is probably produced by effects other than aberrations, such as retinal stray light scattered at the choroid. ${ }^{46,47}$ Retinal scattering increases for longer wavelengths due to their deeper penetration within the retina and the choroid. ${ }^{48,} 49$

Some previous comparisons of optical quality in IR and green light were based on estimates from double-pass aerial images. We performed a computer simulation to evaluate the contribution to the aerial image spread caused by degradation other than the ocular aberrations and the influence of wavelength on this additional contribution. We simulated LRT double-pass aerial images from the estimated wave aberration function. LRT aerial images are the autocorrelation of the entry (first pass) and exit (second pass) point-spread function. The entry pupil is a narrow incoming Gaussian beam (variance $=0.1034$ $\mathrm{mm}$ and $0.1332 \mathrm{~mm}$, respectively, for green and IR illumination) and the exit pupil is a $3-\mathrm{mm}$ circular pupil. The entry and exit pupil sizes correspond to the experimental values in the LRT setup. Insets in Fig. 8 show real images and simulated images corresponding to an entry pupil centered at coordinates $(+1.5,-2.6 \mathrm{~mm})$. Fig. $8 \mathrm{~A}$ and B shows experimental and simulated results for green and IR light, respectively, for eye $\# 22$. The plots represent the normalized radial intensity profile of the corresponding real (solid) and simulated (dashed) aerial images. The distance from the peak position to the zero position represents the centroid deviation from the chief ray (which is practically the same for the simulated and real images). The width of the simulated images accounts for the spread caused exclusively by the measured aberrations, whereas the real images are further enlarged by scattering and nonmeasured higher-order aberrations.

The $\mathrm{SH}$ images in Fig. 2C also suggest a larger contribution of scattered light in IR. A crossed-polarization configuration was used, which explains the "polarization-cross" pattern observed in green light illumination. ${ }^{38}$ Green illumination maximizes the light reflected by the photoreceptor outer segments, ${ }^{50}$ which are thought to partly retain polarization. ${ }^{51}$ Light multiply scattered by deeper layers (probably a significant component of the IR images ${ }^{49}$ ) does not retain polarization, and, therefore, the $\mathrm{SH}$ spots will show little polarization-related intensity differences across the image. ${ }^{38}$

The effects mentioned above affect the shape and intensity distribution of the aerial image and are critical in double-pass measurements of the optical quality of the eye. In this technique, modulation transfer function estimates are directly obtained from double-pass aerial images. An appropriate halo subtraction is critical to obtain modulation transfer functions in IR consistent to those measured in green light. ${ }^{36}$ However, reflectometric techniques for wave aberration measurements only rely on centroid deviation computations, which, as we have shown, are not significantly affected by wavelength.

\section{Chromatic Difference of Focus}

The defocus term was significantly different across wavelengths in all but one subject. The mean focus difference between green and IR across subjects was $0.78 \pm 0.29 \mathrm{D}$, close to the shift estimated by the 

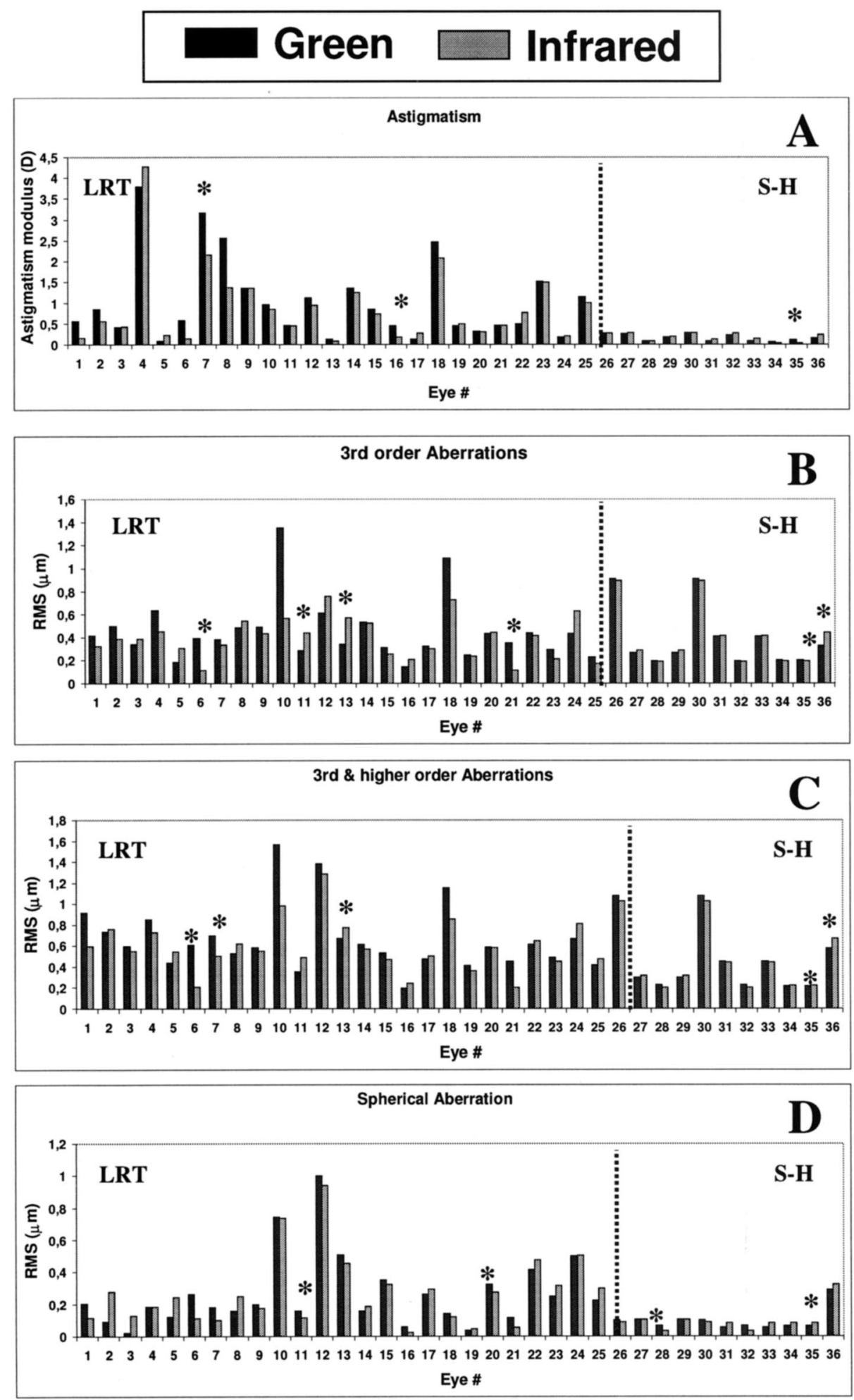

FIGURE 7.

Bar diagrams comparing individual terms (astigmatism and spherical aberrations) and the root mean square wavefront error (RMS) for different orders, obtained with green (black bars) and infrared (gray bars) for all subjects. Eyes \#1 to \#25 were measured with laser ray tracing (LRT), and eyes \#26 to \#36 were measured with a Shack-Hartmann wavefront sensor $(\mathrm{SH})$. Asterisks indicate eyes showing statistically significant differences $(p<0.01)$. 

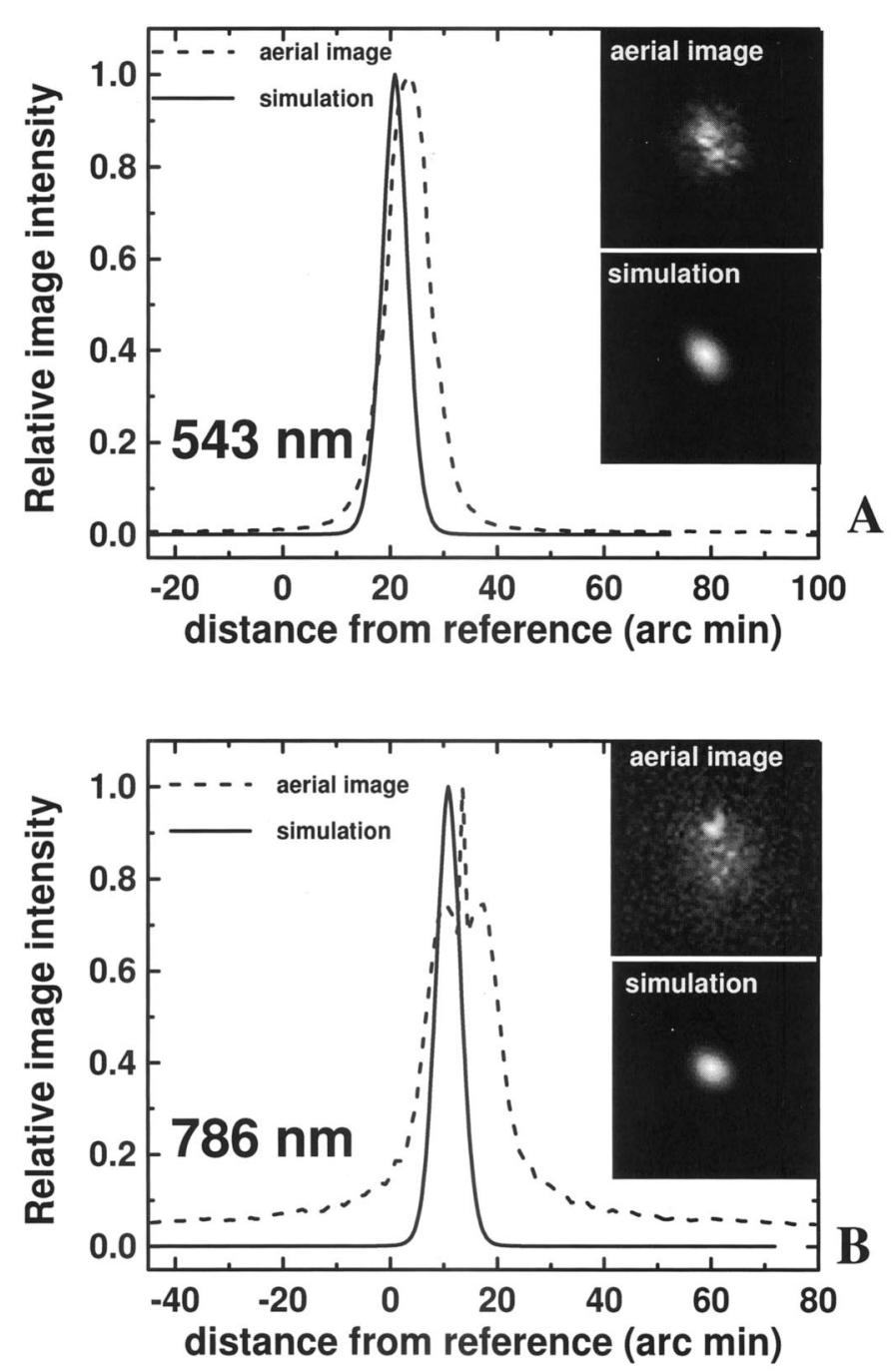

\section{FIGURE 8.}

Experimental and simulated aerial images for green (A) and infrared (B) light, respectively, for eye $\# 22$ and entry pupil at coordinates $(+1.5,-2.6$ $\mathrm{mm})$. The image on the upper left corner of the plot is the aerial image obtained experimentally, and the image below is the aerial image simulated from measured aberrations as the autocorrelation of first- and second-pass point-spread functions. The plots represent the normalized radial intensity profile of the corresponding real (dashed) and simulated (solid) aerial images. The distance to zero position represents the centroid deviation from the chief ray. The width of the simulated images accounts for the spread caused exclusively by the measured aberrations, whereas that of the real images also includes other effects, such as scattering and nonmeasured higher-order aberrations, together with the measured aberrations.

linear fitting shown in Fig. 7 (0.72 D). This value agrees well, within the inherent variability, with the chromatic focus shift predicted by the Indiana chromatic reduced eye model. ${ }^{52}$

$$
\begin{array}{r}
R_{E}=633.46 \times\left[\left(\frac{1}{\left(\lambda_{G}-214.102\right)}-\frac{1}{\lambda_{I R}-214.102}\right]\right. \\
=0.82 \mathrm{D}(1)
\end{array}
$$

where $\lambda_{\mathrm{G}}=543 \mathrm{~nm}$ and $\lambda_{\mathrm{IR}}=787 \mathrm{~nm}$ (mean between IR wavelength used was $786 \mathrm{~nm}$ for LRT and $788 \mathrm{~nm}$ for SH).

Thibos et al. ${ }^{52}$ obtained the parameters of the eye model by fitting experimental data for a range of wavelengths between 400 $\mathrm{nm}$ and $700 \mathrm{~nm}$ and using Cornu's expression for the dependence of the index of refraction with wavelength. Equation 1 agrees well with experimental data in the literature for wavelengths up to 760 $\mathrm{nm}$ (close to the wavelength used in this study), with variations close to the intersubject variability in our sample. ${ }^{52}$ Whether this expression for the LCA still holds for longer wavelengths used in some commercial SH systems (i.e., $830 \mathrm{~nm}$ ) remains to be studied. Typically, Cornu's equation fails beyond the visible, and other expressions $^{53}$ should be used.

It has been frequently argued that differences in the retinal layer where light is reflected may cause differences between manifest refraction and retinoscopy. ${ }^{54,55}$ Charman et al. ${ }^{56}$ and Williams et al. ${ }^{45}$ for red light, and later López-Gil and Artal ${ }^{36}$ for near IR light showed that the differences between subjective and reflectometric focus were negligible and concluded that reflection contributing to the central core of the point-spread function occurred within the photoreceptor layer. Our results, based on the Zernike defocus term of wave aberration reflectometric estimates, also support this conclusion. The focus shift that we found is slightly lower than the chromatic shift prediction (by $0.10 \mathrm{D}$ ), which is consistent with a reflection plane behind the photoreceptor layer. However, this shift is of the order of the measurement error $(0.12 \mathrm{D}$ for green light and $0.08 \mathrm{D}$ for IR light on average) and lower than the intersubject variability $(0.29 \mathrm{D})$. We did not find any particular trend for the focus shift in normal, young subjects as a function of refractive error (coefficient of correlation, $r=0.166, p=0.44$ ). In addition, we did not find any particular difference for the focus shift in eyes with abnormal corneas by laser-assisted in situ keratomileuesis surgery. However, we found that the focus shift for the aphakic eye was much higher than the average (1.7 D). Our population did not sample different age groups homogeneously. However, we found a slight increase of focus shift with age $(r=0.45$, $\mathrm{p}=0.022$ ). The majority of subjects were young or middle-aged (20 to 43 years old), and we could not find an aged-related trend $(\mathrm{r}=0.26, \mathrm{p}=0.2)$.

\section{CONCLUSION}

We have shown the equivalence of high-order aberrations measured in visible or near infrared illumination with LRT and SH, at least within the accuracy of the techniques. The shift in the defocus term was consistent with the shift predicted by chromatic aberration. These results are relevant because typical commercial wavefront-sensing devices use infrared illumination. This wavelength has several advantages over visible illumination: it is more comfortable for the subject, pupil dilation is not essential, and light exposure can be lower due to the higher reflectance of the eye fundus and the better sensitivity of most of the photodetectors at this wavelength. We have shown that despite the longer tails of the aerial images at this wavelength, it can be successfully used in all the tested conditions, including old and surgical eyes.

We also provide an experimental value for the focus shift between near infrared (786 to $788 \mathrm{~nm})$ and green $(543 \mathrm{~nm})$ illumination in two reflectometric aberrometers (LRT and SH). One of the most promising applications of wavefront-sensing devices is their use as sophisticated autorefractometers. They are now being 
applied for use in refractive surgery to guide ablation with the aim of compensating both low- (second order) and high-order (third and higher) aberrations. An accurate transformation of the IR estimates of spherical error into visible wavelengths is crucial to determine the actual correction that should be applied. We have shown that Thibos's chromatic reduced eye model equation is a valid expression to predict focus shift for our wavelength. However, for longer wavelengths, there is no evidence of the validity of this equation, and new expressions for the refractive index and chromatic difference of refraction may need to be developed. In addition, we found that discrepancies can occur in aphakic eyes and that there might be age-dependent corrections to Equation 1. Several reports in the literature found differences in the LCA's of aphakic eyes ${ }^{57}$ and pseudoaphakic eyes ${ }^{58}$ with respect to normal eyes. Possible age-related changes of LCA have been a matter of controversy. ${ }^{59-62}$ Although much of these refractive discrepancies are small, their magnitude can be comparable to the higher-order aberrations, and therefore accurate predictions of spherical errors for visible light from IR measurements are important.

\section{ACKNOWLEDGMENTS}

This study was supported by grants CAM 08.7/0010./2000 from Comunidad Autónoma de Madrid, Spain and BFM 2002-02638, Ministerio de Ciencia y Tecnologia, Spain to S. Marcos, TCI98-0925-C02-01 from the Ministerio de Educación y Cultura, Spain to R. Navarro, CONACyT 150238 predoctoral fellowship, Mexico to D. Lara-Saucedo and CAM 03/0101/1999 to L. Llorente.

We thank S. Barbero for his valuable help with experimental measurements. E. Moreno Barriuso contributed in the early stages of this study. We are indebted to C. Dainty and the Photonics Optics Group at Imperial College for allowing the use and modifications of the wavefront sensor and to $Y$. Tsang from City University for his invaluable help during the experimental sessions. Received April 30, 2002; revision received July 26, 2002.

\section{REFERENCES}

1. He JC, Burns SA, Marcos S. Monochromatic aberrations in the accommodated human eye. Vision Res 2000;40:41-8.

2. Atchison DA, Collins MJ, Wildsoet CF, Christensen J, Waterworth MD. Measurement of monochromatic ocular aberrations of human eyes as a function of accommodation by the Howland aberroscope technique. Vision Res 1995;35:313-23.

3. McLellan JS, Marcos S, Burns SA. Age-related changes in monochromatic wave aberrations of the human eye. Invest Ophthalmol Vis Sci 2001;42:1390-5.

4. Artal P, Berrio E, Guirao A, Piers P. Contribution of the cornea and internal surfaces to the change of ocular aberrations with age. J Opt Soc Am (A) 2002;19:137-43.

5. Navarro R, Moreno E, Dorronsoro C. Monochromatic aberrations and point-spread functions of the human eye across the visual field. J Opt Soc Am (A) 1998;15:2522-9.

6. Collins MJ, Wildsoet CF, Atchison DA. Monochromatic aberrations and myopia. Vision Res 1995;35:1157-63.

7. Marcos S, Moreno-Barriuso E, Llorente L, Navarro R, Barbero S. Do myopic eyes suffer from larger amount of aberrations? In: Thorn F, Troilo D, Gwiazda J, eds. Myopia 2000: Proceedings of the VIII International Conference on Myopia. Boston: The New England College of Optometry, 2000:118-21.

8. Barbero S, Marcos S, Merayo-Lloves J, Moreno-Barriuso E. Validation of the estimation of corneal aberrations from videokeratography in keratoconus. J Refract Surg 2002;18:263-70.
9. Schwiegerling J. Cone dimensions in keratoconus using Zernike polynomials. Optom Vis Sci 1997;74:963-9.

10. López-Gil N, Marín JM, Castejón-Mochón J, Benito A, Artal P. Ocular and corneal aberrations after corneal transplantation. Invest Ophthalmol Vis Sci 2001;42:S529.

11. Munson K, Hong X, Thibos LN. Use of a Shack-Hartmann aberrometer to assess the optical outcome of corneal transplantation in a keratoconic eye. Optom Vis Sci 2001;78:866-71.

12. Seiler T, Kaemmerer M, Mierdel P, Krinke HE. Ocular optical aberrations after photorefractive keratectomy for myopia and myopic astigmatism. Arch Ophthalmol 2000;118:17-21.

13. Moreno-Barriuso E, Lloves JM, Marcos S, Navarro R, Llorente L, Barbero S. Ocular aberrations before and after myopic corneal refractive surgery: LASIK-induced changes measured with laser ray tracing. Invest Ophthalmol Vis Sci 2001;42:1396-403.

14. Marcos S, Barbero S, Llorente L, Merayo-Lloves J. Optical response to LASIK surgery for myopia from total and corneal aberration measurements. Invest Ophthalmol Vis Sci 2001;42:3349-56.

15. Marcos S. Aberrations and visual performance following standard laser vision correction. J Refract Surg 2001;17:S596-601.

16. MacRae SM, Schwiegerling J, Snyder R. Customized corneal ablation and super vision. J Refract Surg 2000;16:S230-5.

17. Navarro R, Moreno-Barriuso E, Bará S, Mancebo T. Phase-plates for wave aberration compensation in the human eye. Optics Lett 2000; 25:236-8.

18. Hofer H, Chen L, Yoon GY, Singer B, Yamauchi Y, Williams DR. Improvement in retinal image quality with dynamic correction of the eye's aberrations. Optics Express 2001;8:631-3. Available at: http:// www.opticsexpress.org/oearchive/source/31887.htm. Accessed August 28, 2002.

19. Fernandez EJ, Iglesias I, Artal P. Closed-loop adaptive optics in the human eye. Optics Lett 2001;26:746-8.

20. Burns SA, Marcos S, Elsnser AE, Bará S. Contrast improvement for confocal retinal imaging using phase correcting plates. Optics Lett 2002;27:400-2.

21. Navarro R, Losada MA. Aberrations and relative efficiency of light pencils in the living human eye. Optom Vis Sci 1997;74:540-7.

22. Moreno-Barriuso E, Marcos S, Navarro R, Burns SA. Comparing laser ray tracing, the spatially resolved refractometer, and the Hartmann-Shack sensor to measure the ocular wave aberration. Optom Vis Sci 2001;78:152-6.

23. He JC, Marcos S, Webb RH, Burns SA. Measurement of the wavefront aberration of the eye by a fast psychophysical procedure. J Opt Soc Am (A) 1998;15:2449-56.

24. Webb RH, Penney CM, Thompson KP. Measurement of ocular wavefront distortion with a spatially resolved refractometer. Appl Optics 1992;31:3678-86.

25. Mierdel P, Krinke HE, Wiegand W, Kaemmerer M, Seiler T. Measuring device for determining monochromatic aberration of the human eye. Ophthalmologe 1997;94:441-5.

26. Walsh G, Cox MJ. A new computerised video-aberroscope for the determination of the aberration of the human eye. Ophthalmic Physiol Opt 1995;15:403-8.

27. MacRae S, Fujieda M. Slit skiascopic-guided ablation using the Nidek laser. J Refract Surg 2000;16:S576-80.

28. Liang J, Grimm B, Goelz S, Bille JF. Objective measurement of wave aberrations of the human eye with the use of a Hartmann-Shack wave-front sensor. J Opt Soc Am (A) 1994;11:1949-57.

29. Liang J, Williams DR. Aberrations and retinal image quality of the normal human eye. J Opt Soc Am (A) 1997;14:2873-83.

30. Wyszecki G, Stiles WS. Color Science: Concepts and Methods, Quantitative Data and Formulae. New York: Wiley, 1982. 
31. Delori FC, Burns SA. Fundus reflectance and the measurement of crystalline lens density. J Opt Soc Am (A) 1996;13:215-26.

32. van den Berg TJ. Light scattering by donor lenses as a function of depth and wavelength. Invest Ophthalmol Vis Sci 1997;38:1321-32.

33. Hofer HJ, Porter J, Williams DR. Dynamic measurement of the wave aberration of the human eye. Invest Ophthalmol Vis Sci 1998;39: S203.

34. Marcos S, Burns SA, Moreno-Barriusop E, Navarro R. A new approach to the study of ocular chromatic aberrations. Vision Res 1999; 39:4309-23.

35. Rynders MC, Navarro R, Losada MA. Objective measurement of the off-axis longitudinal chromatic aberration in the human eye. Vision Res 1998;38:513-22.

36. Charman WN, Jennings JA. Objective measurements of the longitudinal chromatic aberration of the human eye. Vision Res 1976;16: 999-1005.

37. Lopez-Gil N, Artal P. Comparison of double-pass estimates of the retinal-image quality obtained with green and near-infrared light. J Opt Soc Am (A) 1997; 14:961-71.

38. Lopez-Gil N, Howland HC. Measurement of the eye's near infrared wave-front aberration using the objective crossed-cylinder aberroscope technique. Vision Res 1999;39:2031-7.

39. Marcos S, Diaz-Santana L, Llorente L, Dainty C. Ocular aberrations with ray tracing and Shack-Hartmann wave-front sensors: does polarization play a role? J Opt Soc Am (A) 2002;19:1063-72.

40. American National Standard Institute. American National Standard for the Safe Use of Lasers, Standard Z-136.1-1993. Orlando, FL: The Institute, 1993.

41. Diaz Santana HL, Dainty JC. Single-pass measurements of the wavefront aberrations of the human eye by use of retinal lipofuscin autofluorescence. Optics Lett 1999;24:61-3.

42. Diaz-Santana HL, Dainty JC. Single vs. symmetric and asymmetric double-pass measurements of the wavefront aberration of the human eye. In: Love GD, ed. Proceedings of the Second International Workshop on Adaptive Optics for Industry and Medicine: University of Durham, England, July 12-16, 1999. Singapore: World Scientific, 2000:45-50.

43. Diaz-Santana HL. Wavefront sensing in the human eye with a ShackHartmann sensor. 2000. PhD Thesis. London: Imperial College of Science Technology and Medicine.

44. Moreno-Barriuso E, Navarro R. Laser ray tracing versus HartmannShack sensor for measuring optical aberrations in the human eye. J Opt Soc Am (A) 2000;17:974-85.

45. Thibos LN, Applegate RA, Schwiegerling JT, Webb R. Standards for reporting the optical aberrations of eyes. In: Lakshminarayanan V, ed. OSA trends in optics and photonics. Vision Science and Its Applications, Vol 35. OSA Technical Digest Series. Washington, DC: Optical Society of America, 2000:232-44.

46. Williams DR, Brainard DH, McMahon MJ, Navarro R. Double-pass and interferometric measures of the optical quality of the eye. J Opt Soc Am (A) 1994;11:3123-35.

47. Westheimer G, Liang J. Influence of ocular light scatter on the eye's optical performance. J Opt Soc Am (A) 1995;12:1417-24.

48. Westheimer G, Campbell FW. Light distribution in the image formed by the living human eye. J Opt Soc Am 1962;52:1040-4.

49. Elsner AE, Burns SA, Hughes GW, Webb RH. Reflectometry with a scanning laser ophthalmoscope. Appl Optics 1992;31:3697-710.

50. Gorrand JM, Alfieri R, Boire JY. Diffusion of the retinal layers of the living human eye. Vision Res 1984;24:1097-106.

51. Elsner AE, Burns SA, Weiter JJ, Delori FC. Infrared imaging of sub-retinal structures in the human ocular fundus. Vision Res 1996; 36:191-205.

52. Thibos LN, Ye M, Zhang XX, Bradley A. The chromatic eye: a new reduced-eye model of ocular chromatic aberration in humans. Appl Optics 1992;31:3594-600.

53. Herzberger M. Colour correction in optical systems and a new dispersion formula. Optica Acta 1969;5:197-215.

54. Millodot M, O'Leary DJ. On the artifact of retinoscopy and chromatic aberration. Am J Optom Physiol Opt 1980;57:822-4.

55. Millodot M, O'Leary D. The discrepancy between retinoscopic and subjective measurements: effect of age. Am J Optom Physiol Opt 1978;55:309-16.

56. Charman WN. Some sources of discrepancy between static retinoscopy and subjective refraction. Br J Physiol Opt 1975;30:108-18.

57. Millodot $\mathrm{M}$. The influence of age on the chromatic aberration of the eye. Albrecht Von Graefes Arch Klin Exp Ophthalmol 1976;198: 235-43.

58. Negishi K, Ohnuma K, Hirayama N, Noda T. Effect of chromatic aberration on contrast sensitivity in pseudophakic eyes. Arch Ophthalmol 2001;119:1154-8.

59. Howarth PA, Zhang XX, Bradley A, Still DL, Thibos LN. Does the chromatic aberration of the eye vary with age? J Opt Soc Am (A) 1988;5:2087-92.

60. Mordi JA, Adrian WK. Influence of age on chromatic aberration of the human eye. Am J Optom Physiol Opt 1985;62:864-9.

61. Morrell A, Whitefoot HD, Charman WN. Ocular chromatic aberration and age. Ophthalmic Physiol Opt 1991;11:385-90.

62. Calver RI, Cox MJ, Elliott DB. Effect of aging on the monochromatic aberrations of the human eye. J Opt Soc Am (A) 1999;16: 2069-78.

Lourdes Llorente

Instituto de Óptica "Daza de Valdés" Consejo Superior de Investigaciones Cientificas Serrano, 121, Madrid, 28006

Spain

e-mail: lourdes.lle@io.cfmac.csic.es 